F-actin caused by the expression of progerin is, at least partly, relevant to HGPS.

References: 1, Simon DN et al., Nucleus, 2010; 2, Yamazaki S et al., Histochem Cell Biol, 2016; 3, Kubben $\mathrm{N}$ et al., Cell, 2016.

doi: http://dx.doi.org/10.7124/bc.000A07

\section{T-2. Activation of NF-kappaB differentially affects the proliferation rate of different non- small cell lung carcinoma cell lines}

\author{
Dmitri Tentler, Ekaterina Lomert, Ksenia \\ Novitskaya \\ Institute of Cytology, Russian Academy of Sci- \\ ences, Saint Petersburg, Russia \\ dtentler@mail.ru
}

Activity of NF-kappaB transcription factor is usually associated with stimulation of the proliferation potential of cancer cells. On the contrary, our preliminary data suggest that sustained activation of NF-kapp has an opposite effect on certain cell lines. Our research is aimed to dissect mechanisms behind the pro- and anti-proliferative effects of NF-kappaB in non-small cell lung carcinoma (NSCLC) cell lines. Methods: To avoid pleiotropic effects of extracellular stimuli, like TNF or EGF growth factors, we established NSCLC cell lines H1299 and H23 with constant overexpression of the RELA gene, which encodes the primary activation subunit of NF-kappaB. Expression analysis of target genes with qRT-PCR confirmed that H1299/RelA and H23/RelA cell lines maintained enhanced transcriptional activity of the exogenous RelA/p65. The rate of Proliferation, colony formation ca- pacity, cell cycle, apoptosis rate, cellular senescence and expression of genes controlling the cell cycle were investigated. Results: We found that RELA overexpression suppressed proliferation of the H1299 cell culture. The RELA expression level and NF-kappaB transcriptional activity demonstrated inverse correlation with the proliferation rate. Similar results were obtained with the colony formation assay. The cells with high RELA expression had reduced ability to form individual colonies. We than tested a direct involvement of RelA/p65 in the cell cycle control or induction of apoptosis but neither cell cycle nor apoptosis rate were affected. Similarly, the expression analysis of genes involved in regulation of cell cycle and apoptosis (p21/ WAF1, PUMA, BAX) did not reveal any difference. However, the SA-b-gal staining indicated that H1299/RelA had higher rate of cellular senescence comparing to the parental cell line. To test whether the negative impact of NFkappaB activation is common for other NSCLC cell lines, we analyzed effects of RELA overexpression in another NSCLC cells, H23. In contrast to H1299 cells, sustained NF-kappaB activation in $\mathrm{H} 23$ cells led to an enhanced proliferation rate. Further investigations of possible mechanisms behind the differences in NFkappaB activation between the $\mathrm{H} 1299$ and $\mathrm{H} 23$ cell lines are in the progress. Conclusions: We demonstrated that constant NF-kappaB activation might either suppress or stimulate proliferation of different NSCLC cells. The effect does not involve cell cycle control or apoptosis but may implicate cellular senescence. Considering that $\mathrm{H} 1299$ and $\mathrm{H} 23$ cells lines differ in p53 status, further investigation of NF-kappaB may reveal novel aspects of NF-kappaB/interplay in NSCLC. 
The work was supported by the Russian Government Program for the Recruitment of the leading scientists into the Russian Institutions of Higher Education 14. W03.31.0029.

doi: http://dx.doi.org/10.7124/bc.000A00

\section{T-3. Optimization of in vitro model for analysis of tumor cell migration dynamics}
A. O. Kravchenko ${ }^{1,2}$, V. R. Ko- sach $^{1}$, K. A. Shkarina ${ }^{1}$, I. V. Zaiets ${ }^{1,2}$, I. O. Tykhonkova $^{1}$, A. I. Khoruzhenko ${ }^{1}$
${ }^{1}$ Institute of Molecular Biology and Genetics, Kyiv, Ukraine, 03143; ${ }^{2}$ Educational and Scientific Center "Institute of Biology and Medicine", Taras Shevchenko National University of Kyiv 64/13, Volodymyrska Str., Kyiv, Ukraine, 01601 tykhonkova@gmail.com NAS of Ukraine 150, Akademika Zabolotnoho Str.,

Migration ability is an important feature of tumor cells. There are several approaches to analyze the dynamics of cancer cell migration in vitro. One of the most perspective and closer to the in vivo conditions is the model of initiation of the cell migration from 3D multicellular spheroids onto growth surface. Aim. Optimization of the model for adequate quantitative characteristics of the tumor cell locomotion during several days. Methods. 2D and 3D MCF-7 cell culture, immunofluorescence analysis, and image analysis using computer software Fiji. Results. Unification of spheroid size allowed avoiding a significant data deviation. The obtained spheroids spread completely for 3 days. The highest migration ratio was observed at the 2nd day. The proliferation level at each of 3-day experiment was the same and did not exceed $3 \%$. The validity of the model was tested after migration inhibition by rapamycin (mTOR signaling inhibitor). Additionally, this model was successfully applied to immunofluorescence analysis, namely investigation of p85S6K1 subcellular localization in moving MCF-7 cells. Conclusions. Double filtration of multicellular spheroids allowed unification of their size, which promotes an adequate interpretation of the migration assay. This model enabled the study of tumor cells migration dynamics and can be further used for the development of anticancer drug.

doi: http://dx.doi.org/10.7124/bc.000A01

\section{U-1. The involvement of DNA damage response pathway in nuclear reorganization during netotic initiation}

Burhan Uyanik $^{1}$, Bogdan B. Grigorash ${ }^{1,2}$, B. Uyanik ${ }^{1}$, Oleg N. Demidov ${ }^{1,2}$

${ }^{1}$ INSERM 1231, University of Burgundy FrancheComte, Dijon, France; ${ }^{2}$ Institute of Cytology RAS, Saint-Petersburg, Russia uyanik.burhan@hotmail.fr

The netotic type of cell death plays an important role in innate immunity and host defense. It is characterized by epigenetic changes in chromatin modifications, nuclear envelope disassembly, chromatin expulsion and formation of extracellular chromatin nets loaded with anti-bactericidal granules containing aggressive proteinase and pro-oxidant enzymes. Recently, it was shown by several groups that this process has a negative impact on tumor progression. Methods: To study 